\title{
Myositis associated graft-versus-host-disease presenting as respiratory muscle weakness
}

\author{
A L Stephenson, I R A Mackenzie, R D Levy, J Road
}

\begin{abstract}
Myositis associated with graft-versus-hostdisease (GVHD) typically presents with proximal muscle weakness, myalgias, and a raised creatinine phosphokinase (CPK) level. We report a case of a 51 year old man who developed respiratory muscle weakness five years after an allogeneic bone marrow transplant for multiple myeloma. His symptoms included tachypnoea, abdominal paradox, and orthopnoea. Pulmonary function tests revealed diminished vital capacity and maximal inspiratory and expiratory pressures. Serum CPK levels were raised and a peripheral muscle biopsy specimen was consistent with GVHD. He improved with immunosuppressive therapy.

(Thorax 2001;56:82-84)
\end{abstract}

Keywords: graft-versus-host-disease; myositis; respiratory muscle weakness

Graft-versus-host disease (GVHD) is a major cause of morbidity and mortality following allogeneic bone marrow transplantation (BMT); $20-50 \%$ of BMT recipients who survive more than 100 days after BMT will develop chronic GVHD. ${ }^{1}$ Although myositis has been described in association with GVHD, it is rare. ${ }^{2}$ It may be the sole manifestation of active GVHD although patients typically have other organ involvement in addition to muscle. ${ }^{3}$ Myositis associated with GVHD presents in a fashion similar to idiopathic myositis with proximal muscle weakness, myalgias or muscle pain, and an increased creatinine phosphokinase (CPK) level. ${ }^{4}$ To our knowledge, there is only one previously published case report of GVHD myositis involving the respiratory muscles. ${ }^{5}$ This differed from our case in that GVHD developed rapidly two months after a donor leucocyte transfusion (DLT) for relapse of acute myeloblastic leukaemia and the DLT was considered a possible cofactor in the myositis. The histopathological features of GVHD myositis typically show degenerating muscle fibres and necrosis with a perimysial monocytic/ lymphocytic infiltrate with a predominance of CD8+ cytotoxic cells. ${ }^{4}$ Response to treatment with prednisone, with or without cyclosporin, is dramatic with marked improvement of weakness within days of initiating treatment.

\section{Case report}

A 51 year old man who was a non-smoker was admitted to the hospital with a three month history of progressive orthopnoea and cough. $\mathrm{He}$ had undergone an allogeneic BMT from a matched sibling donor for multiple myeloma in 1993. His course was complicated by GVHD involving the skin, bronchiolitis obliterans of the lungs, pulmonary embolism, and central nervous system toxoplasmosis. On admission his medications included prednisone $20 \mathrm{mg}$ alternating with $15 \mathrm{mg}$ daily, trimethoprimsulphamethoxazole $20 \mathrm{ml}$ twice daily Saturday and Sunday, clindamycin $300 \mathrm{mg}$ three times daily three times per week, pyrimethamine $25 \mathrm{mg}$ three times per week, leucovorin $5 \mathrm{mg}$ daily, fluticasone $250 \mu \mathrm{g}$ twice daily, and salbutamol four times daily. Clinically he was tachypnoeic, using accessory muscles of respiration as well as having abdominal paradox, particularly in the supine position. He was noted to have slightly decreased peripheral muscle strength in the upper extremities. The deltoid, triceps, and biceps muscles were graded $4 / 5$ while the muscle groups in the lower limbs were normal strength. A chest radiograph did not show evidence of active pulmonary disease. Arterial blood gas tensions showed evidence of hypoventilation with $\mathrm{pH}$ 7.39, $\mathrm{PaCO}_{2} 6.4 \mathrm{kPa}, \mathrm{PaO}_{2} 10.5 \mathrm{kPa}$, and $\mathrm{HCO}_{3}$ $29 \mathrm{mmol} / \mathrm{l}$ on room air. Nocturnal nasal BIPAP was initiated in hospital with symptomatic improvement of his orthopnoea.

Blood tests showed markedly raised CPK levels and pulmonary function tests revealed significantly decreased vital capacity and severely reduced maximum inspiratory and expiratory pressures (table 1) consistent with respiratory muscle weakness. The vital capacity was performed in the upright position and hence probably overestimated the supine vital capacity by at least $30 \% .{ }^{6}$ Chest fluoroscopy showed severely diminished (virtually absent) bilateral diaphragm motion. No increase in diaphragmatic excursion was seen during the sniff test. A computed tomographic scan of the neck and mediastinum did not show any lesion along the expected course of the phrenic nerve to explain his diaphragmatic weakness. Electromyography (EMG) showed positive sharp waves and fibrillation in the left biceps, triceps, infraspinatus, right biceps, diaphragm, and the paraspinal muscles consistent with either 
Table 1 Sequential values for blood tests and pulmonary function tests

\begin{tabular}{|c|c|c|c|c|c|}
\hline & fuly 22 & Aug 11 & Aug 20 & Aug 27 & Oct 7 \\
\hline Immunosuppressive treatment & & & $\begin{array}{l}\text { Prednisone } 60 \\
\text { mg/day (+ CSA, } \\
\text { MMF) }\end{array}$ & & \\
\hline \multicolumn{6}{|l|}{ Blood tests } \\
\hline $\mathrm{CPK}(\mathrm{U} / \mathrm{l})$ & & 946 & & 347 & 60 \\
\hline \multicolumn{6}{|l|}{ Pulmonary function tests } \\
\hline FVC (1) & $2.06(41)$ & $2.39(47)$ & & $3.04(60)$ & $3.42(68)$ \\
\hline $\mathrm{FEV}_{1}(\mathrm{l})$ & $1.40(35)$ & $1.81(45)$ & & $1.92(48)$ & $2.03(50)$ \\
\hline $\mathrm{FEV}_{1} / \mathrm{FVC}(\%)$ & 68 & 76 & & 63 & 59 \\
\hline $\mathrm{MIP}\left(\mathrm{cm} \mathrm{H}_{2} \mathrm{O}\right)$ & $32(31)$ & $49(48)$ & & $59(58)$ & $99(99)$ \\
\hline $\operatorname{MEP}\left(\mathrm{cm} \mathrm{H}_{2} \mathrm{O}\right)$ & $64.5(42)$ & $81(52)$ & & $114(74)$ & $145(94)$ \\
\hline
\end{tabular}

$\mathrm{CPK}=$ creatinine phosphokinase $; \mathrm{FVC}=$ forced vital capacity $\mathrm{FEV}_{1}=$ forced expiratory volume in one second; MIP. MEP = maximum inspiratory and expiratory pressures; $\mathrm{CSA}=$ cyclosporin; $\mathrm{MMF}=$ mycophenolate mofetil.

Values in parentheses are \% predicted.

myositis, axonal degeneration, or neuromuscular junction dysfunction. A muscle biopsy of the left triceps showed evidence of acute and chronic inflammation with associated muscle fibre necrosis (fig 1) The actively necrotic areas were infiltrated by both polymorphic leucocytes and mononuclear cells. Gram stain and culture of the tissue failed to reveal any infectious aetiology.

The results of the investigations were interpreted as indicating an inflammatory myositis involving not only the proximal muscles, but both the inspiratory and expiratory respiratory muscles, particularly the diaphragm. His prednisone was increased to $60 \mathrm{mg}$ daily and one day later he was started on cyclosporin $50 \mathrm{mg}$ twice daily and mycophenolate mofetil (MMF) $1 \mathrm{~g}$ twice a day. His symptoms improved dramatically over the following week and his CPK level normalised. Repeat pulmonary function testing showed improved muscle strength with increased vital capacity and normal inspiratory and expiratory pressures. Lung volumes measured on 27 August (table 1) revealed total lung capacity of 5.511 (77\% predicted), residual volume of 2.471 (117\% predicted), and normal transfer factor. One month after starting treatment he no longer needed nocturnal BIPAP and his orthopnoea had completely resolved.

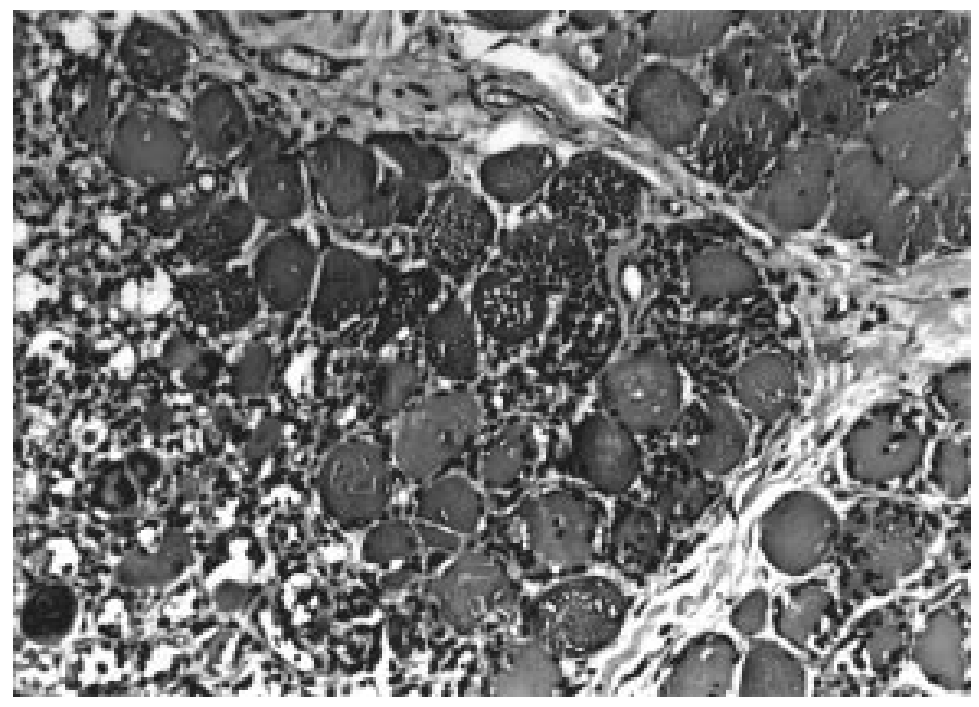

Figure 1 Muscle biopsy specimen of left triceps showing evidence of acute and chronic inflammation associated with muscle necrosis.

\section{Discussion}

Inflammatory myopathy has been seen with chronic GVHD, although involvement of the respiratory muscles is rare with only one case report found in the literature. Five years after BMT our patient developed orthopnoea, general muscle weakness, and increased CPK levels with evidence on clinical examination, pulmonary function testing, and EMG studies of respiratory muscle weakness, particularly of the diaphragm. Although the EMG results are non-specific, they can be seen in inflammatory myopathies caused by GVHD. He responded dramatically to prednisone, cyclosporin, and MMF with resolution of his symptoms, normalisation of his CPK, and improvement of his vital capacity and respiratory muscle strength to the normal range. Interestingly, the spirometric parameters, as well as the inspiratory and expiratory pressures, improved between 22 July and 11 August before his immunosuppression was increased. His corticosteroid inhaler dose was increased during that period as well as his use of $\beta$ agonists, which may have contributed to an improvement in his forced expiratory volume in one second $\left(\mathrm{FEV}_{1}\right)$ and forced vital capacity (FVC). Respiratory muscle strength may have improved following the institution of nocturnal BIPAP which began on 24 July. BIPAP is known to decrease the work of breathing which may have allowed the respiratory muscles to recover partially and hence improve their function.

The muscle biopsy specimen showed evidence of muscle necrosis and an inflammatory infiltrate. The inflammatory cells seen were both neutrophils and monocytes which is somewhat atypical of GVHD myositis. Reported cases have documented a lymphocytic/ monocytic predominant infiltrate on the muscle biopsy specimen. The presence of neutrophils in this case raised the possibility of an infectious process although no organism was cultured. Toxoplasma infection is a recognised risk in the immunocompromised host. Increased toxoplasma IgM levels suggestive of an active infection have been reported in idiopathic polymyositis but not in GVHD myositis. $^{7}$ Our patient had a history of CNS toxoplasmosis and was on suppressive therapy with clindamycin, pyrimethamine, and leucovorin. No toxoplasma organism was cultured in blood, cerebrospinal fluid, or muscle biopsy, making active infection very unlikely in this patient. In addition, increasing the steroid dose would have worsened infection if present, but instead his clinical picture improved. Other possible causes for his weakness include steroid myopathy, but this seems unlikely given his clinical response to augmented steroids.

In conclusion, myositis associated with GVHD typically affects the proximal muscles, but it can also affect the respiratory muscles and cause significant morbidity in this population. The one published case report of GVHD involving the respiratory muscles resulted in respiratory failure and death soon after a donor leucocyte transfusion. ${ }^{5}$ It is possible that the donor $\mathrm{T}$ cells were responsible for causing severe GVHD. Our case illustrates that BMT patients are at risk for respiratory muscle 
involvement with GVHD and that this can be an insidious process occurring many years after the BMT. Furthermore, once muscle atrophy occurs, it has been suggested that the muscle may not recover despite increased immunosuppression and control of the inflammatory process. For this reason, a high clinical index of suspicion is needed for accurate diagnosis so that appropriate treatment can be instituted promptly.

1 Vogelsang GB, Wagner JE. Graft versus host disease. Hematol Oncol Clin North Am 1990;4:625-40. 2 Parker P, Chao NJ, Ben-ezra J, et al. Polymyositis as a mani-
festation of chronic graft-versus-host-disease. Medicine festation of chroni

3 Nelson KR, McQuillen MP. Neurologic complications of graft-versus-host-disease. Neurol Clin 1988;6:389-403.

4 Parker PM, Openshaw H, Forman SJ. Myositis associated with graft-versus-host-disease. Curr Opin Rheumatol 1997; 9:513-9.

5 Oshima Y, Takahashi S, Nagayama H, et al. Fatal GVHD demonstrating an involvement of respiratory muscle following donor leukocyte transfusion. Bone Marrow Transplant 1997:19:737-40.

6 Green M. Respiratory muscle testing. Bull Eur Physiopathol Green M. Respiratory

7 Nagid SK, Kagen LG. Serologic evidence for acute toxoplasmosis in polymyositis/dermatomyositis: Increased frequency of specific antitoxoplasma IgM antibodies. Am $\mathcal{F}$ Med 1983;75:313-20.

\section{LETTERS TO THE EDITOR}

\section{CT scanning in lung cancer}

We read with interest the article by Laroche $e t$ al on the cost effectiveness of performing computed tomographic (CT) scanning before fibreoptic bronchoscopy (FOB) in patients with suspected endobronchial carcinoma. Compared with the group in which FOB was performed blind to the result of the CT scan, in the group in which the CT result was available before FOB was performed the FOB was obviated in $24 \%$ of patients, a higher proportion of the FOBs were diagnostic, and the initial invasive investigation was more frequently diagnostic. The authors do not specify which $\mathrm{CT}$ appearances determined that $\mathrm{FOB}$ should not be undertaken.

We have recently performed a complementary study ${ }^{2}$ evaluating the ability of CT scanning to predict in which patients with an abnormal chest radiograph and high clinical suspicion of bronchial carcinoma FOB is likely to provide a positive histological diagnosis. In distinction to Laroche et al, we excluded patients with pulmonary collapse but included patients with peripheral lesions.

We identified four CT features which predict a positive FOB. In descending order of accuracy these were: (1) a segmental or larger airway leading to the mass; (2) an endobronchial component; (3) situation of the mass within $4 \mathrm{~cm}$ of the origin of the nearest lobar bronchus; and (4) an ill defined edge to the mass. A "gestalt" assessment of the likelihood of positive FOB was slightly more accurate than any individual factor. When two or more predictive factors were present, FOB was positive in $>70 \%$ of our selected series. Of interest, proximity of the lesion to the hilum per se was a poor predictor of positive FOB.

We agree with Laroche et al that CT scanning should be performed before FOB in most patients with an abnormal chest radiograph and a high clinical suspicion of bronchial carcinoma. Our study clarifies how the CT appearances can be used to select those patients in whom FOB is unlikely to be diagnostic and should probably not be performed.

H K BUNGAY
R J O DAVIES
F V GLEESON
Department of Radiology,
Churchill Hospital,
Headington,
Oxford OX3 7Lf, UK
Correspondence to: Dr F V Gleeson
1 Laroche C, Fairburn I, Moss H, et al. Role of
computed tomographic scanning of the thorax
prior to bronchoscopy in the investigation of
suspected lung cancer. Thorax 2000;55:359-63.
2 Bungay HK, Pal CR, Davies CWH, et al. An
evaluation of computed tomography as an aid
to diagnosis in patients undergoing broncho-
scopy for suspected bronchial carcinoma. Clin
Radiol 2000 (in press).

[Editors' note: The authors of the paper were given the opportunity to respond to this letter but declined to do so.]

\section{"Opportunist" mycobacterial infection}

With reference to the letter by Drs Davies and Grange $^{1}$ in the August edition of Thorax and the response to this by Drs Campbell and Ormerod $^{2}$ representing the committee that prepared the guidelines to which this letter refers, ${ }^{3}$ and despite the hope of Drs Campbell and Ormerod that arguments about the nomenclature of mycobacterial grouping would have been laid to rest by now, I believe I can see why this issue remains topical.

Drs Davies and Grange argue that the term "environmental mycobacteria" is the preferred term and should be universally adopted. However, as recently as 1994 D Davies ${ }^{4}$ suggested that, in recognising it is time the world agreed on what to call these mycobacteria, the term "non-tuberculous mycobacteria" should be used and not "environmental mycobacteria" as he now suggests in his letter. ${ }^{1}$ With high profile, expert clinicians changing their minds and advice, it is hardly surprising that guidance is somewhat unreliable and continuously being sought, especially by more junior members of staff. I suspect that, in view of this, it will be some time before this issue is finally laid to rest.

GARY PORTER-JONES Respiratory Specialist Nurse, Department of Respiratory Medicine, North West Wales NHS Trust, Ysbyty Gwynedd Bangor, Gwynedd LL57 2PW, gary.porter-jones@nww-tr.wales.nhs.uk

1 Davies PDO, Grange JM. "Opportunist" mycobacterial infections. Thorax 2000;55:722

2 Campbell IA, Ormerod LP. "Opportunist" mycobacterial infections. Thorax 2000;55:722. 3 Subcommittee of the Joint Tuberculosis Committee of the British Thoracic Society. Management of opportunist mycobacterial infections: Joint Tuberculosis Committee guidelines 1999. Thorax 2000;55:210-8.

4 Davies PDO. Infection with non-tuberculous mycobacteria. Br f Hosp Med 1994;52:375-7.

\section{Tuberculosis in Cuba}

I congratulate Dr Marrero and colleagues on the excellent paper published recently in Thorax about their experience with tuberculosis in Cuba from 1962 to $1997^{1}$ and consider that many developing countries, together with some developed countries like ours, should imitate their methods and their political commitment to a national tuberculosis programme. However, I wish to raise some questions and to comment on some points.

I was pleasantly surprised at the considerable improvement in the case finding from 1993 onwards. From 1983 to 1993 the number of new cases of tuberculosis per year ranged from 514 to 789 with $85.5-91 \%$ smear positive patients (similar to the period since 1962). In 1994 there was a sudden increase to 1617 cases (from 789 cases in 1993) with a significant decrease in the percentage of smear positive patients to $56 \%$ (from $91 \%$ in 1993). This sharp fall was maintained up to 1997 . The authors consider that the expansion of the case definition by the World Health Organization in 1994 to include "clinical symptoms and radiological features suggestive of tuberculosis but with negative sputum smears and negative culture" may have been a significant factor, but in my opinion this is already included in the 
definition "pulmonary tuberculosis with negative sputum smear and positive or negative culture". I would argue that improved case finding as a result of the evident increase in the number of patients with respiratory symptoms studied (from 62370 in 1993 to 179493 in 1997) and in the number of contacts per case of tuberculosis studied (from 4.8 in 1993 to 12.5 in 1997) led to the sudden decrease in the rate of smear negative patients. This decrease is more appropriate and credible for a country in which the distribution of tuberculosis cases by age is similar to that found in industrialised nations, ${ }^{23}$ a fact that Dr Marrero et al point out in their paper.

$$
\begin{array}{r}
\text { A M GARCIA-ZAMALLOA } \\
\text { Service of Internal Medicine, } \\
\text { Hospital of Mendaro, } \\
20850 \text { Gipuzkoa, } \\
\text { Spain }
\end{array}
$$

hmzama@hmen.osakidetza.net

1 Marrero A, Caminero JA, Rodriguez R, et al. Towards elimination of tuberculosis in a low income country: the experience of Cuba, 1962-97. Thorax 2000;55:39-45.

2 Caminero JA. Medidas basicas para el control de la tuberculosis en una comunidad. Med Clin (Barc) 1994;102:67-73.

3 Dye Ch, Scheele S, Dolin P, et al (WHO Global Surveillance and Monitoring Project ). Global burden of tuberculosis: estimated incidence prevalence and mortality by country. $7 A M A$ 1999;282:677-86.

AUTHORS' REPLY We appreciate the comments by Dr Garcia-Zamalloa on our paper on the control of tuberculosis in Cuba. We stated in the discussion that "better case finding and the change in the case definition may explain the increase in the period 1992-1994". The improvement in case finding is related to the comments by Dr Garcia-Zamalloa concerning the better detection of patients with respiratory symptoms and contacts per case studied. However, in 1994 there was a change in the case definition in Cuba because, before this year, patients with clinical symptoms and radiological features suggestive of tuberculosis who were smear and culture negative were not included in the register of the programme. This change and the better case finding also influenced the decrease in the percentage of smear positive cases.

J A CAMINERO

A MARRERO

International Union Against Tuberculosis and Lung
Disease,

Servicio de Neumologia, University Hospital "Dr Negrin", Las Palmas de Gran Canaria, Spain

jcaminer@separ.es

\section{Birth weight and adult lung function in China}

In a study published in Thorax in 1997 Stein and colleagues ${ }^{1}$ showed that birth weight was associated with adult lung function in an Indian population. We have carried out a similar analysis in a Chinese cohort of 59 men and 61 women born in Hong Kong in 1967 and followed up in 1997. This Hong Kong study has been described recently and has shown a significant inverse association between size at birth and adult blood pressure. ${ }^{2}$ Spirometric tests were performed according to the American Thoracic Society's criteria to assure the quality. The same equipment (Sensor Medics 2200) was used in all subjects. Data were analysed by multiple linear regressions; adult height, smoking status, and sex were included as covariates.

The mean birth weight was $3.1 \mathrm{~kg}$ (range 2.3-4.1). Table 1 shows that babies of low birth
Table 1 Mean (SD) FEV,$F V C$, and $F E V_{1} / F V C$ ratio at 30 years of age, adjusted for sex, height at 30 years, and smoking (vs non-smoking) according to birth weight $(n=120)$

\begin{tabular}{lrlll}
\hline Birth weight $(\mathrm{kg})$ & \multicolumn{1}{l}{$n$} & $F E V_{1}(\mathrm{l})$ & $F V C$ (l) & FEV $V_{1} / F V C(\%)$ \\
\hline$\leqslant 2.50$ & 4 & $3.09(0.29)$ & $3.64(0.33)$ & $84.7(6.2)$ \\
$2.50-2.74$ & 18 & $3.23(0.46)$ & $3.69(0.47)$ & $87.9(8.9)$ \\
$2.75-2.99$ & 26 & $3.38(0.32)$ & $3.85(0.50)$ & $89.1(5.6)$ \\
$3.00-3.24$ & 36 & $3.34(0.44)$ & $3.79(0.53)$ & $88.8(5.4)$ \\
$3.25-3.49$ & 19 & $3.34(0.41)$ & $3.81(0.54)$ & $88.4(6.0)$ \\
$3.50-3.74$ & 11 & $3.32(0.28)$ & $3.76(0.36)$ & $88.5(4.7)$ \\
$\geqslant 3.75$ & 6 & $3.36(0.37)$ & $3.68(0.47)$ & $91.0(4.1)$ \\
p for trend * & & 0.36 & 0.87 & 0.30 \\
\hline
\end{tabular}

^ Using birth weight as a continuous variable.

weight $(\leqslant 2.5 \mathrm{~kg})$ had lower mean values of forced expiratory volume in one second $\left(\mathrm{FEV}_{1}\right)$, forced vital capacity (FVC), and $\mathrm{FEV}_{1} / \mathrm{FVC}$ ratio at the age of 30 years, adjusted for sex, adult height, and smoking. However, there was no significant trend (each $\mathrm{p}>0.1$ ). It can be seen from the findings presented by the Indian study that the previously reported associations between birth weight and $\mathrm{FEV}_{1}$ in men and between birth weight and FVC in both sexes were largely attributable to a lower lung function in subjects with a birth weight of less than $5 \mathrm{lb}\left(2.27 \mathrm{~kg}\right.$; table 2 in Stein et $\left.a l^{1}\right)$. In that study there was no obvious trend among the other subjects.

While we fully acknowledge the limitation of a relatively small sample, we believe that it is important to report statistically negative findings and to compare them with the previous Asian study. That our study has shown a statistically significant relation between size at birth and adult blood pressure also suggests that the numbers were sufficiently large to reveal a clinically important association. Having considered findings from our study and the previous one we suggest that, while low birth weight may be associated with a reduced lung function in adults, variation in birth weight among subjects with a normal birth weight did not appear to be relevant.

Y B CHEUNG J P E KARLBERG L LOW Department of Paediatrics M IP Department of Medicine, University of Hong Kong, Hong Kong jpekarl@hkucc.hku.hk

Correspondence to: Professor J P E Karlberg

1 Stein CE, Kumaran K, Fall CHD, et al. Relation of fetal growth to adult lung function in South India. Thorax 1997;52:895-9.

2 Cheung YB, Low LCK, Osmond C, et al. Fetal growth and early postnatal growth are related to blood pressure in adults. Hypertension 2000 (in press).

3 American Thoracic Society. Standardization of spirometry. Am f Respir Crit Care Med 1995; 152:1107-36.

\section{Hyperventilation syndrome}

The editorial by Dr Gardner ${ }^{1}$ on controversial aspects of the hyperventilation syndrome (HVS) refers to our study ${ }^{2}$ in the same issue of Thorax. This study showed that patients with HVS have an accentuated increase in ventilation as a response to change in body position from supine to standing. The editorial was a valuable addition to this difficult subject. We feel, however, that the interpretation of our paper in the editorial did not quite match the purpose or the message of the original study.

We agree with Dr Gardner that the definitions of HVS in the literature are unfortunately variable. Dr Gardner suggests that the term HVS should be abandoned and that efforts should be made to find the initiating and sustaining causes of hyperventilation. This is also our strategy, so the subjects in our study underwent a comprehensive set of cardiopulmonary examinations. In clinical practice, however, the aetiology of hyperventilation often remains unknown and the only finding may be a disproportionate ventilatory pattern with resulting hypocapnia and alkalosis which may (at least partly) be the sustaining cause of the symptoms. Why would we not call the disorder HVS? An alternative label would be "unknown dyspnoea" which does not assure the patient of the benign nature of the disorder. Dr Gardner suggests that our subjects "fit into a classification of dyspnoea and air hunger with secondary intermittent hyperventilation". This classification would probably include the whole spectrum of differential diagnoses of dyspnoea and it is not justified, when several diagnostic procedures have been performed, to exclude cardiopulmonary diseases when the hyperventilatory component of the disorder has been objectively documented. In contrast to Dr Gardner, we also believe that the finding of hyperventilation may be of importance when the initiating cause is known, since not all patients with cardiopulmonary diseases have such a tendency. The disproportionate compensatory mechanisms of ventilation and the resulting hypocapnia may therefore be a sign of inherent susceptibility to hyperventilation and may be responsible for part of the patients' symptoms.

In our study ${ }^{2}$ the organic causes of dyspnoea were excluded as far as possible by specialist care in a university hospital. For the assessment of eventual panic disorder, symptom criteria described by the World Health Organization for research were used. Contrary to what Dr Gardner states in his editorial, the diagnosis of HVS in the study was based on episodic symptoms typical of HVS and documented respiratory alkalosis (with concomitant hypocapnia) in the arterial blood during such an episode. We consider this to be close to the original definition by Geisler et al. ${ }^{3}$ The approach to the definition of HVS was therefore physiological and unambiguous. As this was clearly described in the study, it is difficult to understand the confusion by Dr Gardner when he claims that the diagnosis was made in the presence of normal $\mathrm{PaCO}_{2}$. The measured orthostatic response which was the object of investigation is another matter and should not be confused with the process of diagnosis.

Finally, we would point out that the main purpose of our paper was to describe the accentuated breathing response to orthostatic changes in patients with HVS. We hope that this finding will add to the knowledge of the causes and mechanisms of hyperventilation called for by Dr Gardner. Contrary to the repeated claim in his editorial, ${ }^{1}$ our intention was not to present the orthostatic test as a "diagnostic criterion for HVS" nor as a basis for its diagnosis - assessment of these patients 
is much more complicated. Instead, we stated that measurements of pulmonary gas exchange during the orthostatic test "are/may be useful in the clinical evaluation of patients with hyperventilation disorders". However, based on our results and clinical experience over 15 years, we believe that this test is, indeed, helpful as part of the diagnostic evaluation of HVS.

L P MALMBERG K TAMMINEN A R A SOVIJÄRVI Laboratory of Clinical Physiology, Helsinki University Central Hospital, Haartmaninkatu 2 FIN 00290 Helsinki, Finland anssi.sovijarvi@hus.fi

Correspondence to: Professor A Sovijarvi

1 Gardner W. Orthostatic increase of respiratory gas exchange in hyperventilation syndrome. Thorax 2000;55:257-9.

2 Malmberg LP, Tamminen K, Sovijärvi ARA. Orthostatic increase of respiratory gas exOrthostatic increase of respiratory gas ex-
change in hyperventilation syndrome. Thorax change in hyperve

3 Geisler L, Herberg D, Thorspecken R. Diagnose und Therapie des Hyperventilationssyndroms. Fortschr Med 1965;83:463-6.

\section{Supplemental oxygen in COPD}

I read with interest the study by Garrod et al on the use of supplemental oxygen during pulmonary rehabilitation in patients with COPD with exercise hypoxaemia. ${ }^{1}$ Most studies on supplemental oxygen are difficult to interpret because they are either not randomised or placebo controlled. It is therefore important that they have dealt with these problems. Nevertheless, I have some difficulty in interpreting their results. The patients were randomised to either training with air (AT) or oxygen (OT). These two groups were tested without additional gas (test 1 ), with gas (air or oxygen) before training (test 2), and with gas after six weeks of training (test 3). I have reproduced their data on the shuttle walk test (SWT) and post SWT Borg score (Borg) in table 1.

When they compared AT and OT at test 2 they found that oxygen had a significant effect on the SWT (mean difference $27.3 \mathrm{~m}$ ) and on the Borg score (mean difference -0.68 ). Surprisingly, although the difference between OT and AT at test 1 was larger, this difference was reported as insignificant.

If changes in the mean outcome measures (test 2 minus test 1 ) are compared (as they did after six weeks of training), the differences between OT and AT are $-1.7 \mathrm{~m}$ for SWT and 0.02 for the Borg score. The results therefore
Table 1 Acute effect of oxygen compared with air and the effect of training with oxygen compared with training with air

\begin{tabular}{lllll}
\hline & Test 1 & Test 2 & Test 3 & Test 2-Test 1 \\
\hline Oxygen (OT) & 160.0 & 176.4 & 196.4 & 16.4 \\
$\quad$ SWT (m) & 3.83 & 3.52 & 2.52 & -0.31 \\
$\quad$ Borg & 131.0 & 149.1 & 192.7 & 18.1 \\
Air training (AT) & 4.53 & 4.20 & 4.66 & -0.33 \\
$\quad$ SWT (m) & & & & -1.7 \\
$\quad$ Borg & 29.0 & 27.3 & 3.7 & 0.02 \\
Difference (OT-AT) & -0.70 & -0.68 & -2.14 & \\
$\quad$ BWT (m) & & & & \\
\hline
\end{tabular}

depend on the methods used to compare the two groups. The authors need to explain why they compared absolute values when they evaluated the acute effect of oxygen and differences when they evaluated the effect of oxygen during training.

T RINGBAEK Moseskraenten 17, DK-3140 Aalsgaarde, Denmark ringbaek@dadlnet.dk

1 Garrod R, Paul EA, Wedzicha JA. Supplemental oxygen during pulmonary rehabilitation in patients with COPD with exercise hypoxaemia. Thorax 2000;55:539-43.

AUTHORS' REPLY We are grateful for the comments by Dr Ringbaek but, unfortunately, he has confused the data from two separate tables published in our paper.

Test 1 concerns baseline data which was performed to minimise practice effects and was not included in the analysis. Test 2 (176.4 and 149.1) concerns the results of the shuttle walking test (SWT) with an oxygen cylinder and air cylinder in the group as a whole and not separate oxygen training (OT) and air training (AT) groups. This was clearly stated in the legend.

The study was concerned with the additional effect of oxygen on a training programme in patients with severe COPD. We take the point that the results depend on the method used to compare the two groups, as is often the case with statistical analysis. However, change was chosen as a more sensitive outcome as it enables one to take account of baseline variables.

R GARROD

J A WEDZICHA

St Bartholomew's and Royal London School of Medicine and Dentistry, St Bartholomew's Hospital, London EC1A $7 B E$,

f.A.Wedzicha@mds.qmw.ac.uk

\section{BOOK REVIEW}

Handbook of Sleep Medicine. J M Shneerson (Pp 248; £49.50). Oxford, UK: Blackwell Science. 0632051353

This excellent book, written by one of the pioneers of sleep disordered breathing in the UK, provides a broad coverage of disorders of sleep that is especially relevant for respiratory physicians. The particular strength of this book is that it provides fascinating background information on the physiology and pathophysiology of sleep which ideally complements the chapters on more specific respiratory problems such as obstructive sleep apnoea syndrome and disorders of respiratory drive and mechanics. Respiratory physicians who see patients with obstructive sleep apnoea syndrome will invariably get involved in trying to assist those with clear evidence of a sleep disorder but without any apparent common respiratory problems. This book will provide such physicians with a background working knowledge of the differential diagnosis in these circumstances, together with some especially helpful summaries and breakdowns of the investigation and management of abnormal sleep cycles.

The book is also well laid out. I found the layout of the book in twin columns to be physically very accessible, and my only criticism is the frequent use of abbreviations in tables and text which, for the unfamiliar reader, is the only interruption to the flow of ideas in the book. Overall, however, I think this is a highly informative text which I imagine will provide a valuable resource to anyone seeing patients who complain of sleepiness.-JB 